\title{
Development of Rutin Ethosomes for Enhanced Skin Permeation
}

\author{
Anju Dhiman*, Deepika Singh, Kaneez Fatima and Ghazala Zia \\ Department of Pharmaceutical Sciences, Maharshi Dayanand University, Rohtak, Haryana, India
}

\section{Article Info}

\author{
*Corresponding author: \\ Anju Dhiman \\ Department of Pharmaceutical Sciences \\ Maharshi Dayanand University \\ Rohtak-124001, Haryana \\ India \\ E-mail: ad_mdu@rediffmail.com
}

Received: January 27, 2019

Accepted: February 19, 2019

Published: March 1, 2019

Citation: Dhiman A, Singh D, Fatima K, Zia G. Development of Rutin Ethosomes for Enhanced Skin Permeation. Int J Tradit Med Appl. 2019; 1(1): 4-10.

doi: $10.18689 /$ ijtma-1000102

Copyright: (c) 2019 The Author(s). This work is licensed under a Creative Commons Attribution 4.0 International License, which permits unrestricted use, distribution, and reproduction in any medium, provided the original work is properly cited.

Published by Madridge Publishers

\begin{abstract}
Objective: The rationale of the study was to prepare rutin ethosomes using butylated hydroxytoulene as permeation enhancer.

Materials and Methods: Rutin ethosomes using butylated hydroxytoulene (BHT) as permeation enhancer were prepared by cold method. The optimization of rutin ethosomes were done by using $2^{3}$ factorial design with the help of design expert software (version 8.0). The optimized rutin ethosomes formulation were then characterized for vesicular shape, size of the particle and the size distribution by using scanning electron microscopy, zeta potential, entrapment efficiency and stability studies. The percutaneous studies of optimized rutin ethosomes with permeation enhancer were done by a specially designed franz diffusion cell by using pig ear skin and the results were studied using DD solver 8 software.
\end{abstract}

Results: The entrapment efficiency study of optimized rutin ethosomes formulated by using phospholipid concentration of $2.5 \mathrm{~g}, 30 \mathrm{ml}$ ethanol and $1.5 \mathrm{~g} \mathrm{BHT}$, and it was found to be maximum i.e. $76.9 \%$. The negative zeta potential of lipid vesicular system of rutin exhibited $-60.8 \mathrm{mV}$ which indicates a high degree of stability for rutin ethosomes with permeation enhancer. In vitro studies of pure rutin and rutin ethosomes for drug permeation using $\mathrm{BHT}$ as permeation enhancer on pig ear skin suggested that rutin ethosomes using $\mathrm{BHT}$ as permeation enhancer showed rutin skin permeation rate of at the end of $120 \mathrm{~min}$. However, at the end the permeation rate of pure rutin shown to be $120 \mathrm{~min}$.

Discussion: Rutin ethosomes with permeation enhancer were better able to facilitate permeation of rutin into the pig ear skin deeply when compared with pure rutin. So, it can be used as an effective drug carrier for transdermal delivery of rutin.

Conclusion: Rutin ethosomal formulation with permeation enhancer has tremendous potential to serve as a topical drug delivery system due to its enhanced skin permeation rate.

Keywords: Ethosome; Herbals; Permeation enhancer; Rutin; Transdermal.

\section{Introduction}

Rutin, a bioflavanoid has low solubility, less stability, low bioavailability and poor permeation. Rutin ethosomes with permeation enhancer were formulated in order to enhance the permeation of rutin through skin [1]. Permeation enhancers are chemicals that interact with the constituents of the biological membrane to increase the drug flux [2]. Chemical substances known as accelerants and sorption promoters temporarily diminishing the barrier of the skin and hence able to enhance drug flux [3]. The permeability of barrier layers of intestinal cells transiently increases by aromatic alcohols. It happens due to the opening of the tight junctions between these cells creating pores through which even large molecules (macromolecules) may pass by diffusion. An alternative mode of action is enhancement of fluid-phase pinocytosis, resulting in internalisation of bulk fluid together with macromolecules 
within vacuoles, which are transported from one side of the cell to the other [4]. In a study, transdermal patches of apomorphine containing butylated hydroxy toluene as permeation enhancer at concentration $0.1,0.5,1,5$ and 10 weight percent has shown good skin adhesion [5]. In the present study, rutin has been selected as model drug because of its antioxidant potential [6]. In this research attempt, rutin ethosomes with permeation enhancer (butylated hydroxytoulene) has been developed and its skin permeation rate has been studied and compared with pure drug, rutin. Chemical structure of rutin is given in figure 1 .

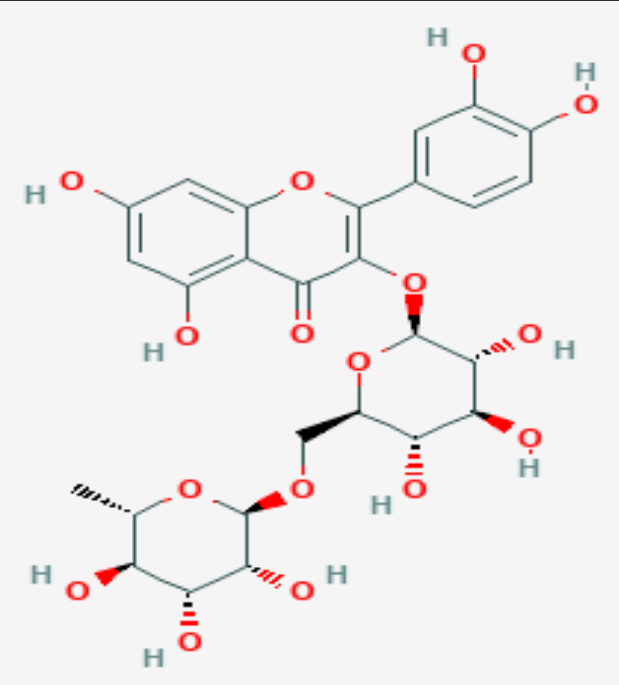

Figure 1. Chemical structure of rutin.

\section{Materials and Methods}

The chemicals were purchased from different sources vi.z. rutin from LobaChemie (Mumbai, India), phosphatidylcholine and BHT from Hi-media (Mumbai, India), and ethanol from Central Drug House (Gujrat, india).

\section{Experimental design}

$2^{3}$ full factorial design for process optimization of rutin ethosomes was used using design expert software (version 8.0).

\section{Preparation of rutin ethosomes}

Cold method was adopted for the preparation of rutin ethosomes. In this method, phospholipid and BHT were taken in a small round bottom flask and solubilised with ethanol containing drug and the mixing was continued by magnetic stirrer. To avoid the evaporation of ethanol round bottom flask was covered. To obtain the ethosome colloidal suspension the distilled water was added slowly with continuous stirring. Then it was kept at room temperature for 30 min under continuous stirring [1].

\section{Characterization of ethosomal system}

Visualization by scanning electron microscopy: Visualization of rutin ethosomes with permeation enhancer was significantly achieved by scanning electron microscopy. Pure rutin and rutin ethosomes were mounted on a clear glass stub. They were air dried and visualized under scanning electron microscope at X10000.

Determination of ethosomal size distribution: The measurement of mean diameter was done by Zeta sizer Nano ZS (Malvern, UK) soon after the preparation and rutin ethosomes was stored at $4 \pm 2^{\circ} \mathrm{C}$. The readings were taken in triplet. Each sample was diluted using de-ionized water and particle size distribution was analyzed.

Determination of zeta potential: Zeta potential is the electrical potential of the vesicles, including its ionic atmosphere [7]. The zeta potential was measured by Zetasizer Nano ZS (Malvern, UK) of the formulated ethanol-containing rutin vesicles with permeation enhancer in distilled water at $20^{\circ} \mathrm{C}$.

Interaction of Phospholipid-ethanol: The phospholipidethanol interaction was assessed by ${ }^{1} \mathrm{H}-\mathrm{NMR}$ using dimethyl sulphoxide as solvent and by IR (Perkin Elmer FTIR spectrophotometer, USA).

Transition temperature: Transition temperature was determined by Mettler-Toledo DSC (Columbus) instrument. The samples were kept in an aluminium pan and crimped them followed by heating the pan under nitrogen flow. Aluminium pan containing same quantity of indium was used as reference. Differential scanning colorimetry scan for rutin and rutinethosome were recorded at heating rate of $10^{\circ} \mathrm{C} /$ min in temperature range of $10^{\circ} \mathrm{C}-300^{\circ} \mathrm{C}$.

Entrapment efficiency: The rutin ethosomes were subjected to entrapment efficiency of rutin with permeation enhancer was determined by ultra centrifugation technique. The total volume of the preparation was studied and $2 \mathrm{ml}$ of the formulation was transferred to $10 \mathrm{ml}$ centrifuge tube. The dilution of the preparation was done by the distilled water up to $5 \mathrm{ml}$ and was centrifuged at 2,000 rpm for 20 minutes using a cooling centrifuge (C-24 BL; Remi Instruments Ltd., Vasai, India) to separate out undissolved drug from the formulation. Ethosomes were separated by ultra centrifugation at 2,000 rpm for $20 \mathrm{~min}$. Supernatant and sediment was recovered and their volume was measured. Following centrifugation, supernatant was removed from pellets and the amount of unentrapped drug was calculated spectrophotometrically at a wavelength $257 \mathrm{~nm}$ using UV visible spectrophotometer (Model-UV-2450, Shimadzu Corp., Kyoto, Japan). The UV calibration curve of rutin obtained was: $y=0016 x$ +0.009 where; $y$ is the concentration of rutin and $x$ is the rutin absorbance, with $R^{2}$ value of 0.98 . The amount of rutin entrapped in the ethosomal formulation was measured as the amount of rutin added during the preparation and its amount recovered in the supernatant.

The entrapment efficiency was determined using the equation (i). Entrapment efficiency $=$ DEDT $\times 100$

Where, DE is the amount of drug in the ethosomal sediment and DT is a theoretical amount of drug used to prepare the formulation

In vitro skin permeation study: The experimental set up for in vitro skin permeation study using franz diffusion cell is shown in figure 2. The ear skin of pig was removed and the pig's ear was obtained from the local abattoir located in Jaipur city. The adhered fat was removed to the dermis side by using isopropyl alcohol and a scalpel. These excised skins was washed with tap water and then phosphate buffer saline $(\mathrm{pH} 7.4)$, dried and placed on aluminium foil, stored in refrigerator and used within two days. The in vitro study for skin permeation of rutin 
ethosomes with BHT and pure rutin was determined using a specially designed Franz glass diffusion cell with effective permeation area of $3.14 \mathrm{~cm}^{2}$ and receptor cell volume of $11 \mathrm{ml}$ as shown in figure 2 . The temperature was set at $37 \pm 0.5^{\circ} \mathrm{C}$. The receptor part contained phosphate buffer saline $(11 \mathrm{ml})$. Excised pig ear skin was mounted between the donor and receptor compartment and $3 \mathrm{ml}$ of preparation was placed in the donor compartment. Samples were withdrawn from the receiver compartment at regular intervals for $120 \mathrm{~min}$ and analyzed spectrophotometrically at $257 \mathrm{~nm}$. The procedure was repeated using rutin ethosomes with BHT as a carrier system. The results were analysed using DDsolver 8 software.

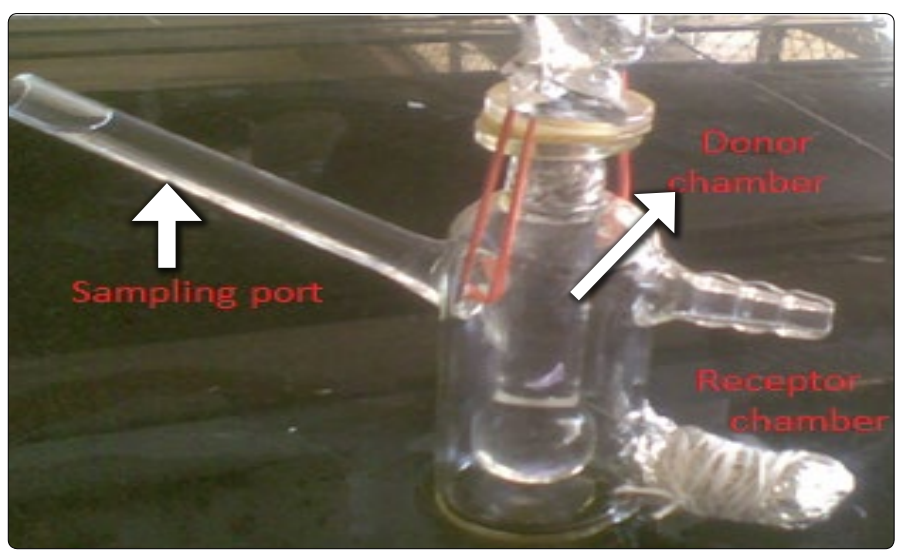

Figure 2. Experimental set up for in vitro skin permeation study using franz diffusion cell.

Stability study: Optimized formulation of rutin ethosomes with $\mathrm{BHT}$ as permeation enhancer was stored at $4^{\circ} \mathrm{C} \pm 2{ }^{\circ} \mathrm{C}$, $40^{\circ} \mathrm{C} \pm 2{ }^{\circ} \mathrm{C} / 70 \pm 5 \% \mathrm{RH}$ and $25^{\circ} \mathrm{C} \pm 2{ }^{\circ} \mathrm{C} / 60 \pm 5 \% \mathrm{RH}$ for about one month and monitored for drug entrapment efficiency, colour change and sedimentation, if any.

\section{Results}

\section{Scanning electron microscopy (SEM)}

The scanning electro.n microscopy showed that acicular crystals of pure rutin and the closed spherical shape were possessed by rutin ethosomes with permeation enhancer as shown in figures $3 a$ and $3 b$ respectively.

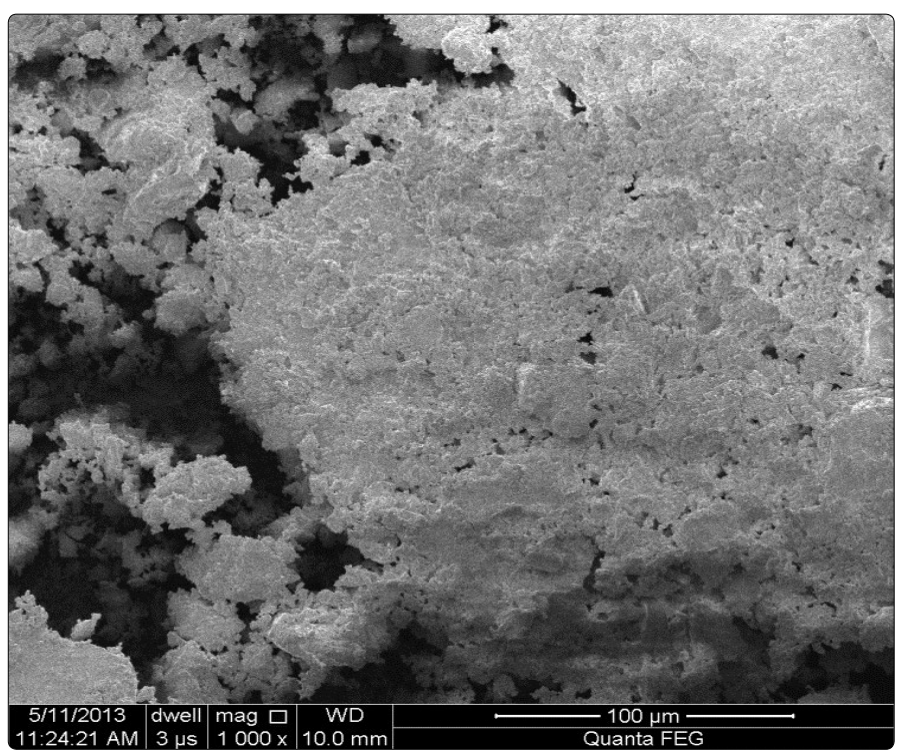

Figure 3a. SEM of rutin.

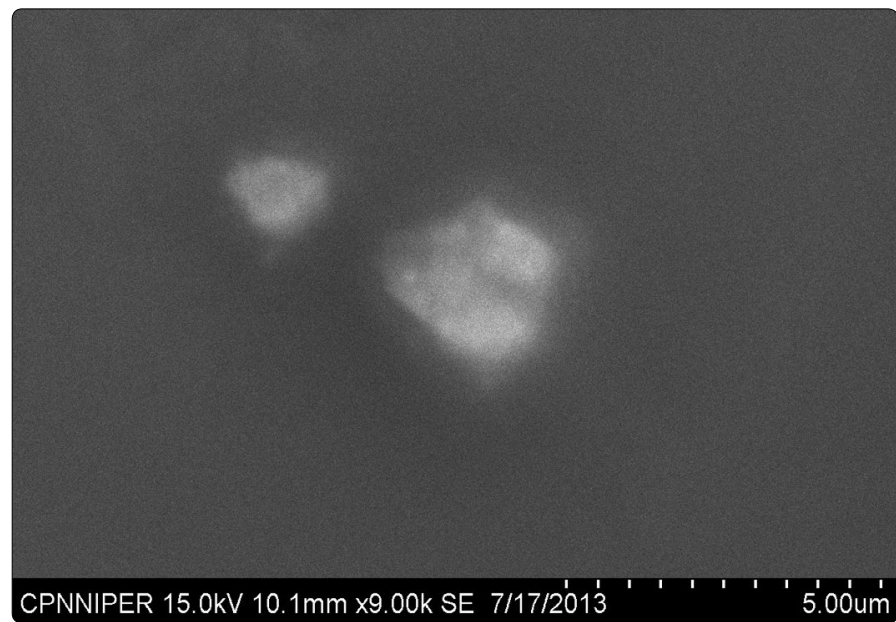

Figure $3 \mathrm{~b}$. SEM of rutinethosome with permeation enhancer, BHT.

\section{Particle size distribution}

The particle size and the distribution analysis of rutin ethosomes with BHT showed a narrow peak which confirming the homogeneous population of rutin ethosomal vesicles size. The rutin ethosomes average size with permeation enhancer was $634.9 \mathrm{~nm}$ and pure rutin was $21.23 \mu \mathrm{m}$ as shown in figures $4 \mathrm{a}$ and $4 \mathrm{~b}$.

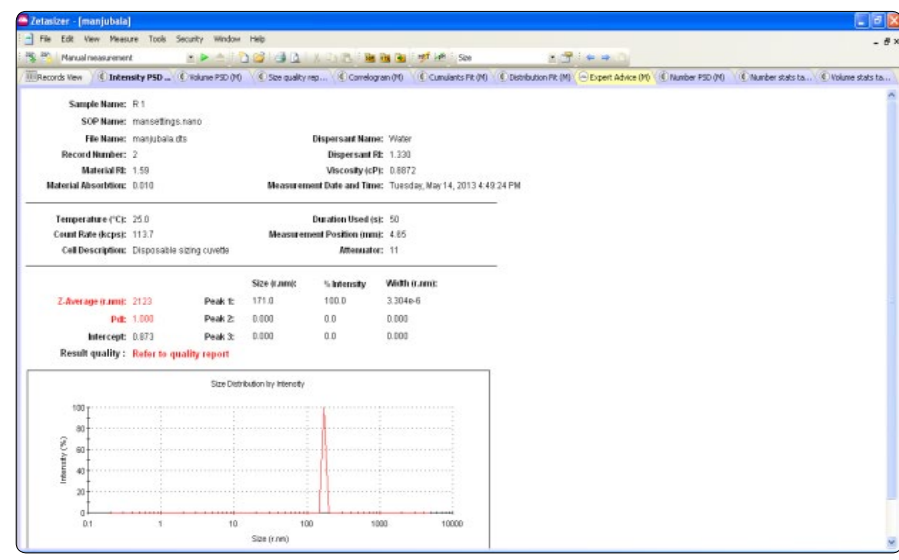

Figure 4a. Size distribution of rutin.

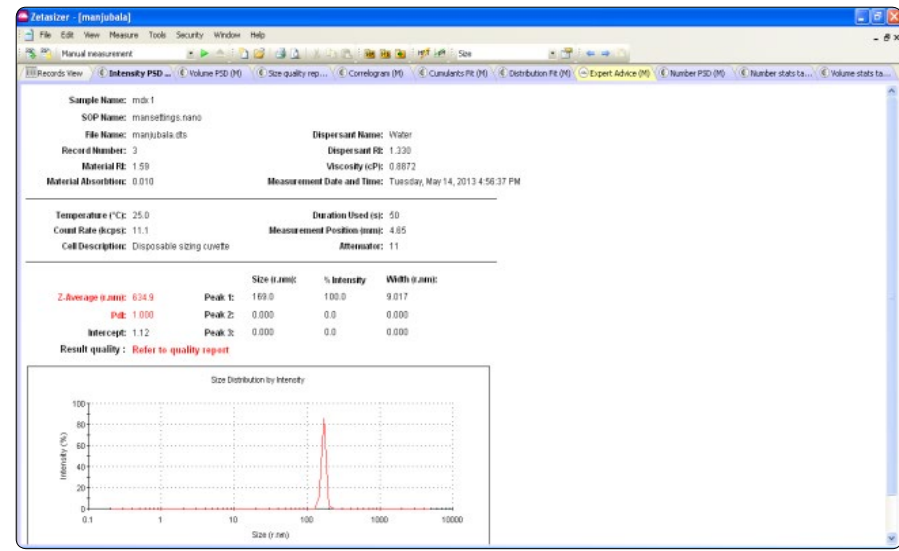

Figure $4 \mathrm{~b}$. Size distribution of rutin ethosomes with permeation enhancer.

\section{Zeta potential}

The rutin ethosomes were investigated for its surfacecharge properties and results revealed that rutin ethosomal preparation have negative charges on their surfaces with Zetapotential value of $-60.8 \mathrm{mV}$ (Figure 5). The Z-potential value highlights a good degree of stability for rutin ethosomes. 


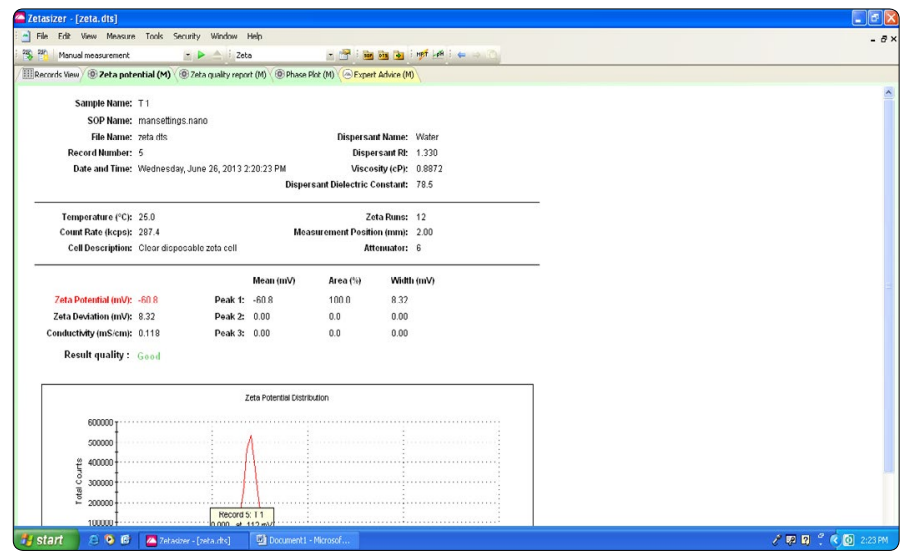

Figure 5. Zeta potential of rutin ethosomes with permeation enhancer, BHT.

\section{Entrapment efficiency}

The different formulation composition consists of total of eight runs according to $2^{3}$ factorial designs. The experimental observation for entrapment efficiencies of all the eight batches are tabulated in table 1. The entrapment efficiency results for all the eight trial combinations were fed into the design expert software to get optimized value for the variable parameters v.i.z. time (min), phospholipid concentration (g) and ethanol ( $\mathrm{ml}$ ) as shown. The entrapment efficiency of rutin ethosomes with BHT was $76.9 \%$ for optimized batch.

The contour plot of optimization of rutin ethosomes for determination of time, phosholipid concentration and ethanol as process parameters is shown in figure 6a. Three-dimensional plot showing the optimized values of time and phospholipid concentration at least possible standard error of design is shown in figure $6 \mathrm{~b}$.

Table $1.2^{3}$ factorial designmatrix of rutin ethosomes with permeation enhancer.

\begin{tabular}{|c|c|c|c|c|}
\hline Experiment & $\begin{array}{c}\text { Phospholipid } \\
(\mathrm{g})\end{array}$ & $\begin{array}{c}\text { Ethanol } \\
(\mathrm{ml})\end{array}$ & $\begin{array}{c}\text { BHT } \\
(\mathrm{g})\end{array}$ & $\begin{array}{c}\text { Entrapment } \\
\text { efficiency (\%) }\end{array}$ \\
\hline 1 & 4 & 10 & 1 & $21.09 \pm 4.37$ \\
\hline 2 & 1 & 10 & 2 & $72.89 \pm 4.77$ \\
\hline 3 & 4 & 50 & 2 & $70.96 \pm 4.55$ \\
\hline 4 & 1 & 50 & 2 & $71.56 \pm 4.55$ \\
\hline 5 & 1 & 10 & 1 & $22.97 \pm 4.14$ \\
\hline 6 & 4 & 10 & 2 & $16.72 \pm 4.88$ \\
\hline 7 & 1 & 50 & 1 & $68.30 \pm 4.23$ \\
\hline 8 & 4 & 50 & 1 & $17.81 \pm 4.75$ \\
\hline
\end{tabular}

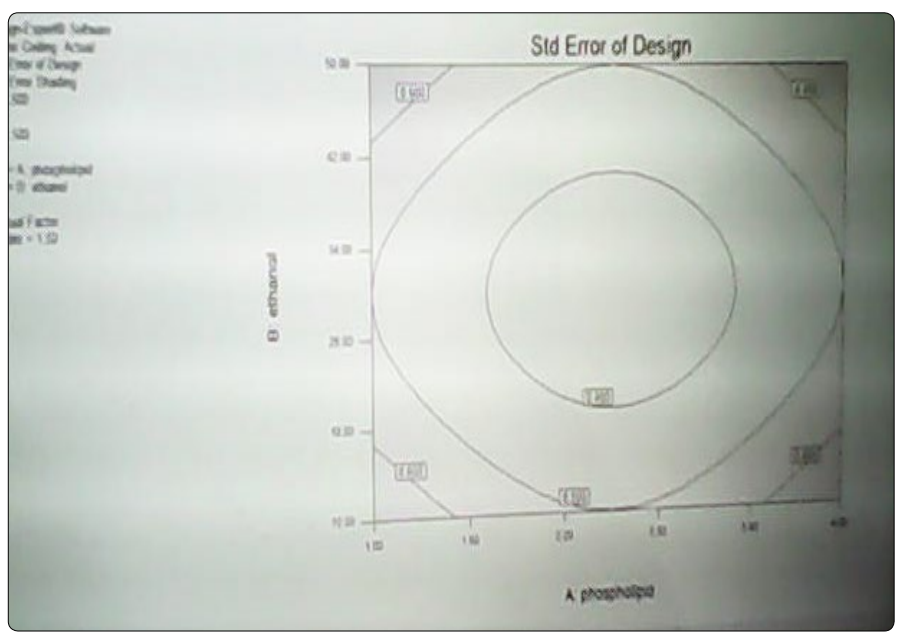

Figure 6a. Contour plot of optimized rutin ethosomes with BHT.

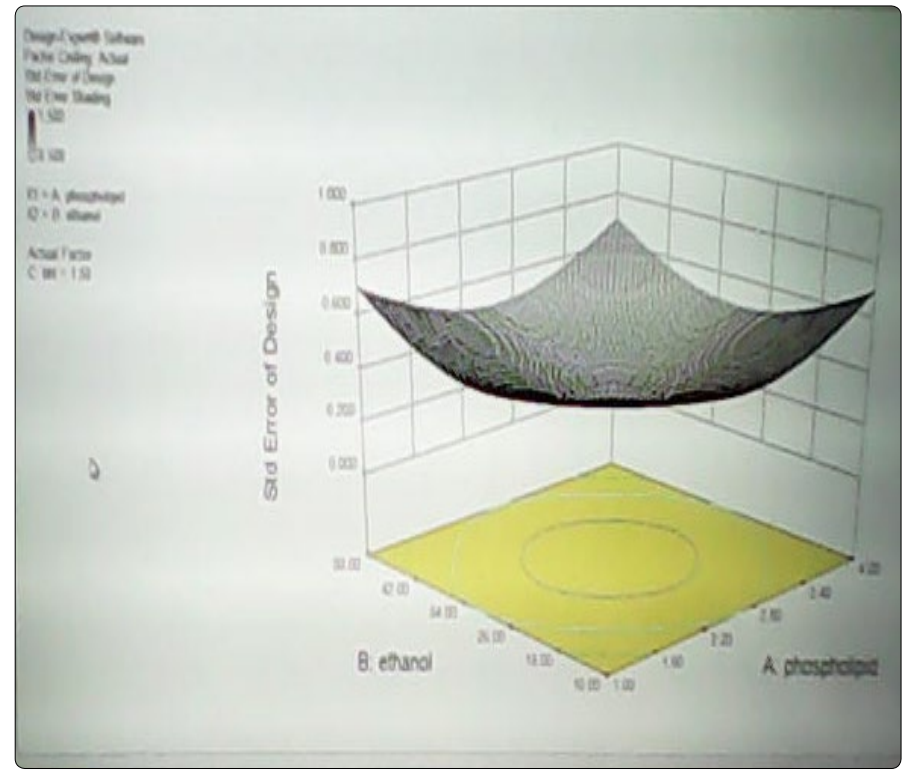

Figure $6 \mathrm{~b}$. Three-dimensional surface view of optimized rutin ethosomes with BHT.

\section{Infrared spectra}

The IR spectra of pure rutin and rutin ethosomes with permeation enhancer are shown in figures $7 \mathrm{a}$ and $7 \mathrm{~b}$ respectively. The IR spectra of rutin showed peak at $1652 \mathrm{~cm}^{-1}$ including $\mathrm{C}=\mathrm{O}$ stretching, aryl ketone), $1503 \mathrm{~cm}^{-1}$ including $\mathrm{C}=\mathrm{C}$ stretching, for phenyl group and bending at $1202 \mathrm{~cm}^{-1} . \mathrm{A}$ peak representing $\mathrm{O}-\mathrm{H}$ functional group also appeared at $1313 \mathrm{~cm}^{-1}$. Other characteristic peak observed was at 1059 $\mathrm{cm}^{-1}$ including C-O-C asymmetric stretching for ether and at $2938 \mathrm{~cm}^{-1}$ due to alkane $\left(\mathrm{CH}_{3}\right.$ asymmetric stretching). IR spectra of rutin ethosomes with $\mathrm{BHT}$ as permeation enhancer showed a peak at $1044 \mathrm{~cm}^{-1}$ representing C-O-C asymmetric stretching for ether group and at $1382 \mathrm{~cm}^{-1}$ which corresponds to $\mathrm{OH}$ stretching. The peak at $1647 \mathrm{~cm}^{-1}(\mathrm{C}=\mathrm{O}$ stretching for aryl ketone) and at $2975 \mathrm{~cm}^{-1}$ due to alkane $\left(\mathrm{CH}_{3}\right.$ assymetric stretching).

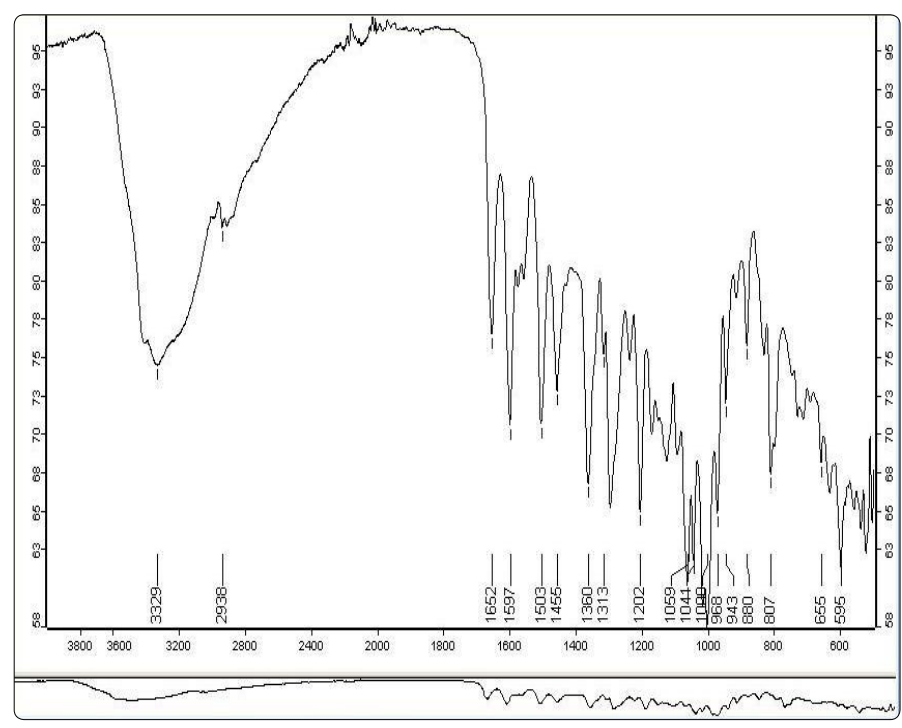

Figure 7a. IR spectra of pure rutin 


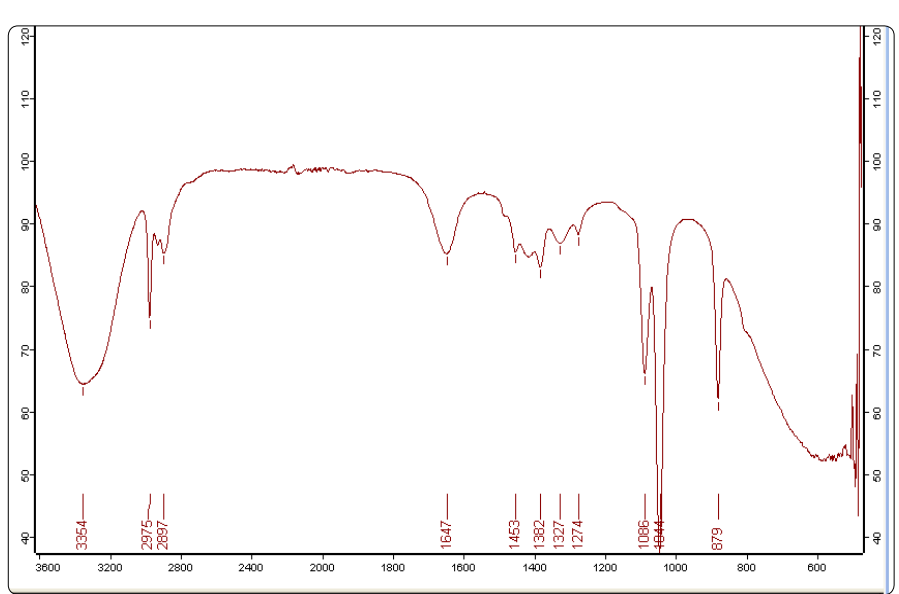

Figure $7 \mathrm{~b}$. IR spectra of rutinethosomes with permeation enhancer, BHT.

\section{NMR spectra}

NMR spectra of rutin and rutin ethosomes are shown in figures $8 \mathrm{a}$ and $8 \mathrm{~b}$ respectively. The spectra of rutin showed peaks at $6.184-6.823(\mathrm{~m}, 5 \mathrm{H}, \mathrm{Ar}), 5.090(\mathrm{~s}, 4 \mathrm{H}, \mathrm{OH}), 5.292(\mathrm{~d}, 1 \mathrm{H},-\mathrm{CH}$ of tetrohydropyran), 4.371 (q, $1 \mathrm{H}, \mathrm{CH}$ of tetrohydropyran), 3.42 ( $\mathrm{t}$, $1 \mathrm{H}, \mathrm{CH}$ of tetrohydropyran) and $2.488(\mathrm{~s}, 1 \mathrm{H}, \mathrm{OH}$ of tetrohydropyran). However, NMR spectra of rutin ethosomes with permeation enhancer showed peak at $4.220(\mathrm{q}, 1 \mathrm{H}, \mathrm{CH}$ of tetrohydropyran), $3.49(\mathrm{t}, 1 \mathrm{H}, \mathrm{CH}$ of tetrohydropyran) and 1.042 $\left(\mathrm{s}, 3 \mathrm{H}_{1} \mathrm{CH}_{3}\right)$ indicating peak shift and changes in peak shape.

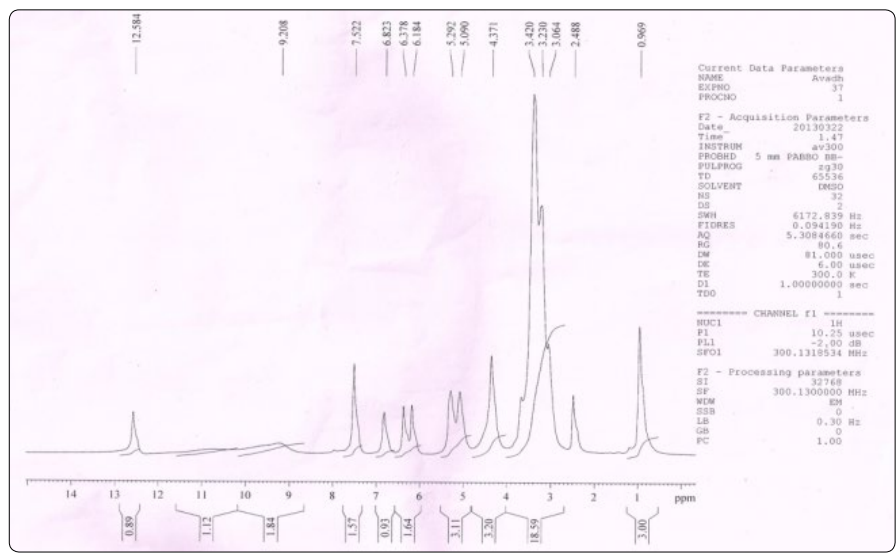

Figure 8a. ${ }^{1} \mathrm{H}-\mathrm{NMR}$ spectra of pure rutin.

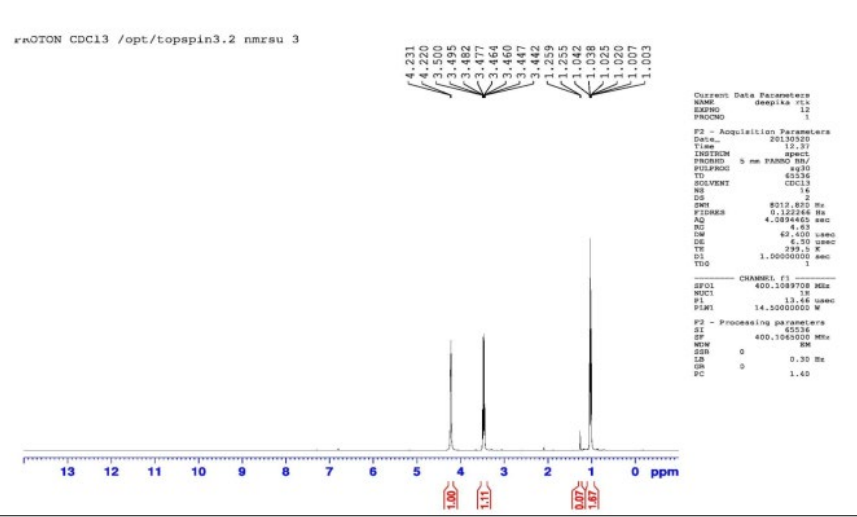

Figure 8b. ${ }^{1} \mathrm{H}-\mathrm{NMR}$ spectra of rutinethosomes with permeation enhancer, BHT.

\section{Differential scanning calorimetry (DSC)}

The melting temperatures of phospholipid in rutin ethosomes with BHT were studied by DSC indicating the degree of phospholipid bilayers fluidity. As per DSC study, the melting point of rutin obtained was $157^{\circ} \mathrm{C}$ while rutin ethosomes melted at $143^{\circ} \mathrm{C}$ (Figures $9 \mathrm{a}$ and $9 \mathrm{~b}$ respectively). The DSC results indicated that the lipid bilayers in rutin ethosomes with permeation enhancer were in a fluid state, representing a soft vesicular structure.

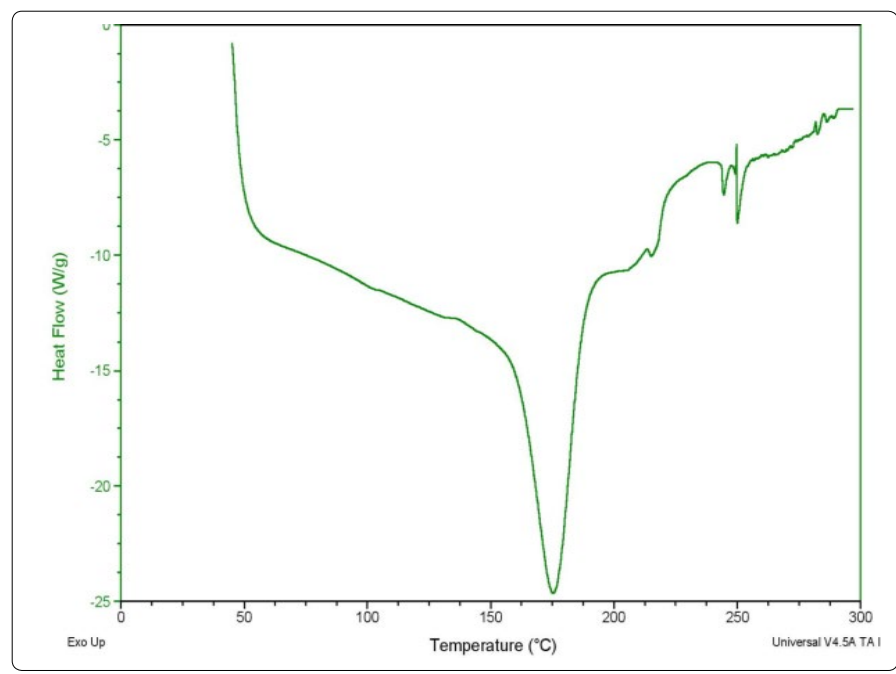

Figure 9a. DSC thermogram of rutin.

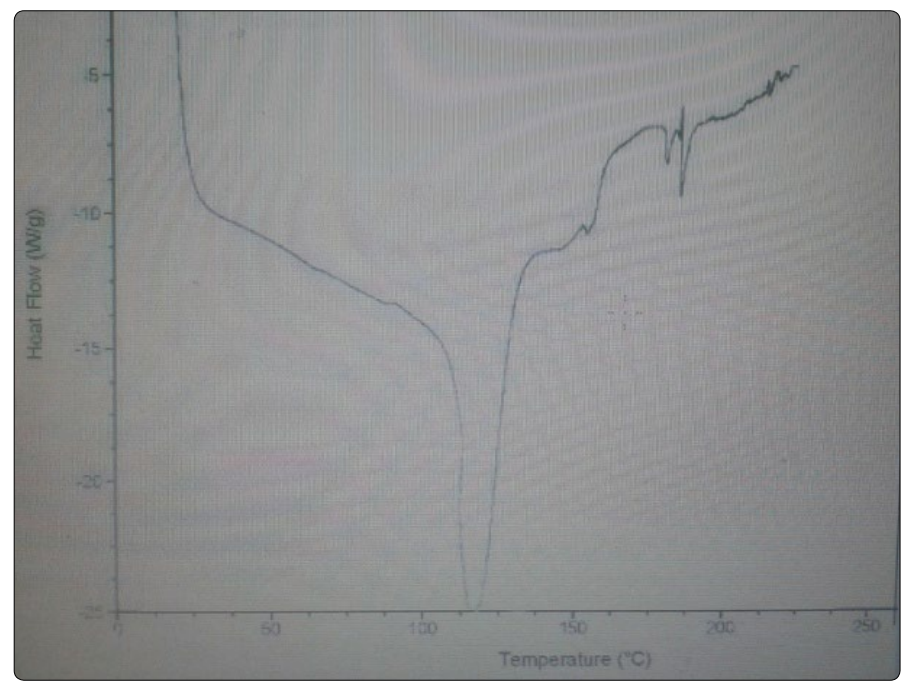

Figure 9b. DSC thermogram of rutinethosomes with BHT.

\section{In-vitro skin permeation study}

Rutin ethosomes showed an increased in-vitro percutaneous permeation of rutin when compared with hydroalcoholic solution of rutin as shown in figure 10 .

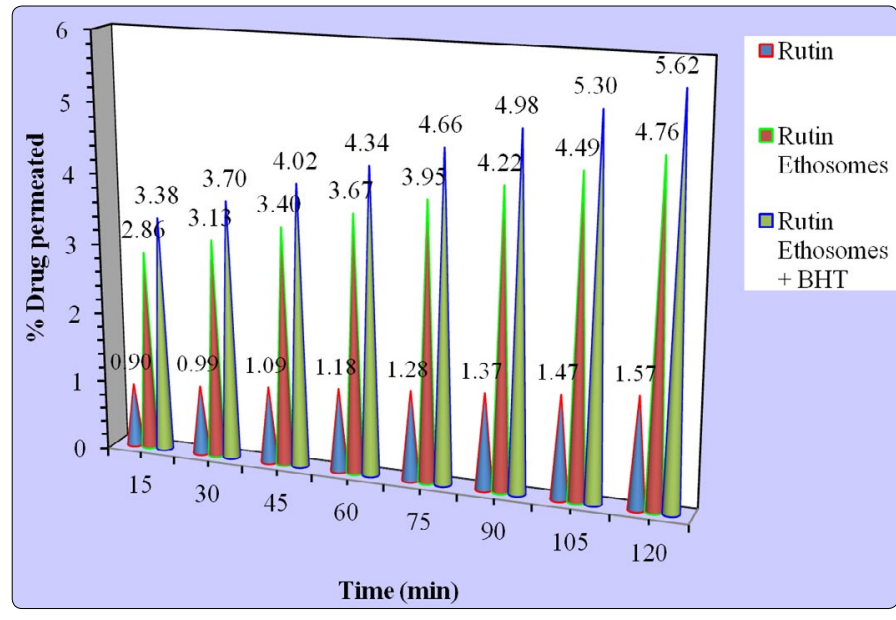

Figure 10. In vitro skin permeation study of rutin, rutin ethosomes and rutin ethosomes with $\mathrm{BHT}$. 


\section{Stability testing}

The accelerated stability testing results of optimized rutin ethosomal formulation with permeation enhancer at different storage conditions are given in table 2 and graph is shown in figure 11. No change in colour and sedimentation was observed in optimized rutin ethosomes with $\mathrm{BHT}$, stored at $4^{\circ} \mathrm{C}$ and $25^{\circ} \mathrm{C}$, however, slight colour change and sedimentation occurred in the formulation stored at high temperature i.e. at $40^{\circ} \mathrm{C}$ at the end of $30^{\text {th }}$ day.

Table 2. Stability study of rutin ethosomes with permeation enhancer.

\begin{tabular}{|c|c|c|c|}
\hline $\begin{array}{c}\text { Time } \\
\text { (days) }\end{array}$ & $\begin{array}{c}\text { Percent entrapment } \\
\left(4^{\circ} \mathrm{C} \pm 2^{\circ} \mathrm{C}\right)\end{array}$ & $\begin{array}{c}\text { Percent entrapment } \\
\left(25^{\circ} \mathrm{C} \pm 2^{\circ} \mathrm{C} / 60 \pm 5 \% \mathrm{RH}\right)\end{array}$ & $\begin{array}{c}\text { Percent entrapment } \\
\left(40^{\circ} \mathrm{C} \pm 2^{\circ} \mathrm{C} / 70 \pm 5 \% \mathrm{RH}\right)\end{array}$ \\
\hline & $76.9 \%$ & $76.9 \%$ & $76.9 \%$ \\
\hline 5 & $76.9 \%$ & $76.8 \%$ & $74.34 \%$ \\
\hline 10 & $76.5 \%$ & $75.75 \%$ & $70.98 \%$ \\
\hline 15 & $76.2 \%$ & $74.84 \%$ & $68.75 \%$ \\
\hline 20 & $75.89 \%$ & $72.89 \%$ & $66.85 \%$ \\
\hline 25 & $75.54 \%$ & $71.67 \%$ & $64.54 \%$ \\
\hline 30 & $74.80 \%$ & $70.56 \%$ & $62.75 \%$ \\
\hline
\end{tabular}

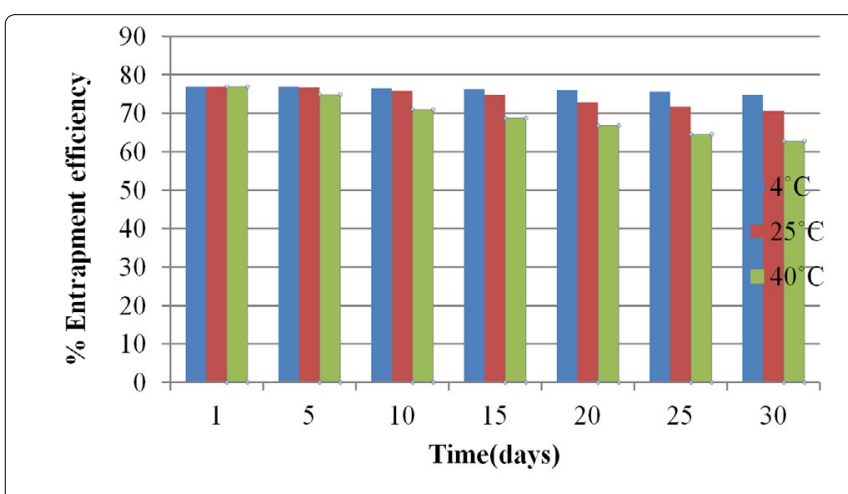

Figure 11. Stability study of rutin ethosomes with permeation enhancer, BHT.

\section{Discussion}

Present study was investigated in vitro skin permeation rate of a novel vesicular system of rutin i.e. rutin ethosomes with permeation enhancer. The cold method is used for the formulation of rutin ethosomes with permeation enhancer and $2^{3}$ factorial design using design expert software (version 8.0). Physicochemical properties such as charge, size, morphology, interaction of phospholipid-ethanol, entrapment efficiency, transition temperature, rate of skin permeation and stability w.r.t. particle aggregation/sedimentation of rutin ethosomes were studied.

Morphological study of rutin was done by SEM. It showed existence of rutin as a yellow coloured acicular crystalline powder with particle size of $21.23 \mu \mathrm{m}$. However, rutin ethosomes with $\mathrm{BHT}$ as permeation enhancer were amorphous in nature, size were found to be small (634 $\mathrm{nm}$ ) and spherically symmetrical.

Zeta potential of rutin ethosomes with BHT showed negative values, which may be due to the presence of ethanol. This negative value of surface charge is related to the size of rutin ethosomal vesicles and a result of high concentration of ethanol. It also highlights high degree of stability for ethosomes [8,9]. Presence of ethanol causes modification in net charge and good degree of stabilization that finally leads to a decrease in mean particle size of vesicles [10]. DSC spectra of rutin ethosomes with BHT showed a decrease in transition temperature value of phospholipids which suggested that the ethosomal bilayers were in a more fluid state that result in soft vesicular configuration [11].

${ }^{1} \mathrm{H}-\mathrm{NMR}$ and IR spectra of rutin ethosomes with BHT confirmed the fluidity effect of ethanol on the ethosomal phospholipid bilayers and phospholipid-ethanol interaction.

The optimized batch of rutin ethosomes with BHT showed the best entrapment efficiency of $76.9 \%$. Higher entrapment efficiency of rutin in rutin ethosomes with permeation enhancer could be due to intrinsic solubility of rutin in ethanol present in the ethosomal core and lipid bilayer. In addition, vesicles containing high ethanol concentration are reported to have thin membranes which correspond to the formation of an interpenetrating hydrocarbon chains thus finally improved the entrapment efficiency of rutin ethosomes [10]. The hydroalcoholic solution of rutin was able to facilitate penetration of rutin deeply into the pig ear skin, but only small amount delivered from the rutin ethosomal formulation. The optimized rutin ethosomes with permeation enhancer were found to be more penetrating with a relatively large quantity of probe deeply into the skin. Investigating the stability of ethosomal vesicles with respect to time is a fundamental criterion for their potential application as transdermal, aggregation or sedimentation occurred during the storage of ethosomal vesicles and many drug delivery system. Various factors such as creaming components of the formulation are responsible for the instability. For this reason, the physicochemical stability of rutine thosomes was investigated [12]. Sedimentation was observed in optimized ethosomal formulation at high temperatures storage conditions. Also, entrapment efficiency of rutin ethosome did not get change significantly even after the storage for one month. At high temperature, there is a decreased in entrapment efficiency that may be due to leakage of drug [13]. The outcomes of the present study suggested that rutin in the form of ethosomal vesicular system with $\mathrm{BHT}$ as permeation enhancer improve in-vitro skin permeation and can be used in the therapies of various of skin ailments.

\section{Conclusion}

In transdermal delivery system, rutin ethosomes have been considered as a possible vasicular carrier as an antiinflammatory agent. This study confirms that ethosomes of flavonoid drugs along with permeation enhancer provide a promising carrier for the delivery of parent drug (rutin, in this case). BHT enhanced accumulation of rutin and optimizes the drug to target in dermal and epidermal sites. This will create new approach for topical application of rutin as antiinflammatory agents.

\section{References}

1. Touitou E, Dayan N, Bergelson L, Godin B, Eliaz M. Ethosomes - novel vesicular carriers for enhanced delivery: characterization and skin penetration properties. J Control Release. 2000; 65(3): 403-418. doi: $10.1016 / \mathrm{s} 0168-3659(99) 00222-9$ 
2. Vaishak MN, Panday A. Assessment of flavonoid release with different permeation enhancers. Int J Pharm Sci Res. 2012; 4(2): 154-156. doi: 10.7439/ijbr

3. Pathan $B$, Shetty $C$. Chemical penetration enhancers for transdermal drug delivery systems. Trop J Pharm Res. 2009; 8(2): 173-179.

4. Roger R. U.S Patent 7. 2006.

5. Durif. U.S Patent 5. 1999.

6. Hendra R, Ahmad S, Oskoueian E, Sukari A, Shukor MY. Antioxidant, antiinflammatory and cytotoxicity of Phaleria macrocarpa (Boerl.) Scheff Fruit. BMC Complement Altern Med. 2011; 11(1). doi: 10.1186/1472-6882-11-110

7. Shang L, Wang Y, Cai S, et al. Partial Dominance, Overdominance, Epistasis and QTL by Environment Interactions Contribute to Heterosis in Two Upland Cotton Hybrids. G3 (Bethesda). 2015; 6(3): 499-507. doi:10.1534/ g3.115.025809

8. Dave V, Kumar D, Lewis S, Paliwal S. Ethosome for enhanced transdermal drug delivery of acelofenac. Int J Drug Deliv. 2010; 2(1): 81-92.
9. Akhtar N, Pathak K. Cavamax W7 Composite Ethosomal Gel of Clotrimazole for Improved Topical Delivery: Development and Comparison with Ethosomal Gel. AAPS Pharm Sci Tech. 2011; 13(1): 344-355. doi: 10.1208/s12249-012-9754-y

10. Zhou Y, Wei Y, Liu H, Zhang G, Wu X. Preparation and in vitro evaluation of ethosomal total alkaloids of Sophora alopecuroides loaded by a transmembrane $\mathrm{pH}$-gradient method. AAPS Pharm Sci Tech. 2010; 11(3): 1350-1358.

11. GodinB, Touitou E. Mechanism of bacitracin permeation enhancement through the skin and cellular membranes from an ethosomal carrier. J Control Release. 2004; 94(2-3): 365-379.

12. Giuliano E, Paolino D, Fresta M, Cosco D. Drug-Loaded Biocompatible Nanocarriers Embedded in Poloxamer 407 Hydrogels as Therapeutic Formulations. Medicines. 2019; 6(1): 1-20. doi: 10.3390/medicines6010007

13. Sathali A, Vijaykumar $M$, Arun K. Formulation and evaluation of diclofenac potassium ethosomes. Indian J Pharm Sci. 2010; 2(4): 82-86. 